\title{
HABITABILIDAD URBANA EN FRACCIONAMIENTOS PERIFÉRICOS EN CIUDAD JUÁREZ CHIHUAHUA, MÉXICO
}

\author{
Urban habitability in peripheral neighborhood in Ciudad Juarez Chihuahua, \\ Mexico
}

\author{
Herrera-Terrazas Luis \\ (Departamento de Arquitectura, Universidad Autónoma de Ciudad Juárez) luis.herrera@uacj.mx \\ Peña Barrera, Leticia \\ (Departamento de Arquitectura, Universidad Autónoma de Ciudad Juárez) Ipena@uacj.mx \\ Ordoñez Hernández, Guillermo \\ (Departamento de Arquitectura, Universidad Autónoma de Ciudad Juárez) gordonez@uacj.mx
}

\begin{abstract}
RESUMEN
La creación de fraccionamientos en zonas periféricas en Ciudad Juárez, alejadas del centro de la ciudad, donde se edifica vivienda mínima para trabajadores, básicamente, de la industria maquiladora, tienen como característica el abandono y deterioro, generándose así problemáticas en las condiciones de habitabilidad, tanto en la vivienda como en lo urbano. El objetivo de la investigación es analizar y conocer la problemática de la localización y construcción de vivienda en lugares alejados del centro de la ciudad. Se pretende conocer la percepción de los habitantes respecto a condiciones de habitabilidad urbana de la zona, así como aspectos de conectividad con otras áreas de la ciudad.
\end{abstract}

Palabras clave: Habitabilidad, normatividad, crecimiento urbano, abandono de vivienda.

Bloque temático: análisis y proyecto territorial.

\begin{abstract}
The creation of neighborhood in peripheral areas in Ciudad Juarez, far from the center of the city, where minimum housing is built for workers, basically, of the maquiladora industry, have the characteristic of abandonment and deterioration, thus generating problems in the conditions of habitability, both in the housing as in the urban. The objective of the research is to analyze and know the problem of the location and construction of housing in places far from the center of the city. It is intended to know the perception of the inhabitants regarding urban habitability conditions in the area, as well as aspects of connectivity with other areas of the city.
\end{abstract}

Keywords: Habitability, regulations, urban growth, abandonment of housing

Topic: analysis and territorial project. 


\section{Introducción}

Ciudad Juárez, Chihuahua, México se localiza en la zona norte de la república, frontera con los Estados Unidos de Norteamérica, lo que le ubica de manera estratégica para el desarrollo económico basado en la producción manufacturera (maquiladoras) desde 1960. Los límites naturales del Río Bravo al norte (demarcación internacional), la sierra de Juárez al poniente y el desierto al suroriente, obligan que el crecimiento de la ciudad, históricamente sea hacia el poniente y oriente del centro fundacional. Sin embargo, las limitantes topográficas de la sierra al poniente y el río al norte, obligan a un desarrollo hacia el sur oriente, esto a partir del desarrollo del Programa de Industrialización Fronteriza (PIF) al inicio de la década de los sesenta, con la industria maquiladora como nueva fuente de desarrollo económico.

La creación de fraccionamientos en zonas periféricas, alejadas del centro de la ciudad, donde se edifica vivienda mínima para trabajadores que laboran en esta industria maquiladora, tienen como característica la ubicación en zonas segregadas de los beneficios urbanos, y con el abandono y deterioro de miles de casas, que han impactado en las condiciones de habitabilidad, tanto en la vivienda como en el contexto social.

La firma de tratados internacionales que permitieron la creación de parques industriales para las empresas maquiladoras, requirió de la concentración de mano de obra del sector obrero u operador; este fenómeno representó un polo de atracción laboral para personas de diferentes partes del país que migraron de otras ciudades con desempleo y vieron una oportunidad de mejorar su situación económica y calidad de vida. En consecuencia este rápido proceso de captación demográfica, implicó que en la ciudad no se contara con las políticas de desarrollo que pudieran absorber la demanda de abasto, educación, salud o de vivienda. En la década de los noventas, el desarrollo habitacional se promueve por medio de la participación de la industria de la construcción, que por un lado continuó con la edificación de naves industriales (a menor escala) y por otro casa-habitación, que mediante la urban sprawl, se generó dispersión y fragmentación del territorio, ocasionando la expansión territorial, la especulación del suelo y problemáticas sociales y económicas que sobrepasaron la capacidad administrativa y reguladora del municipio.

El sur oriente de la ciudad, es el sector con mayor concentración del desarrollo habitacional, mediante fraccionamientos que, para dar abasto a la necesidad de vivienda a un bajo costo, se localizan en propiedades alejadas, son servicios básicos, rodeadas de baldíos y con carencias de equipamiento educativo, recreativo, de salud y cultural, siendo una zona de concentración de familias con nivel socioeconómico bajo.

A pesar de que existen planes reguladores desde 1962 y planes de desarrollo subsecuentes, la ciudad presentó un crecimiento desordenado, mostrando una gran cantidad de vacíos urbanos al interior de la misma, en algunos casos la especulación del suelo dejó espacios sin consolidar con grandes extensiones sin edificar. Esto propicia tener que recorrer grandes distancias para desplazarse de un lugar a otro, la deficiente dotación de servicios e insuficiente equipamiento para el número de habitantes que ahí residen.

El estudio sobre habitabilidad urbana, permite identificar las principales deficiencias de los fraccionamientos periurbanos que presentan características de lejanía y rodeados de vacíos urbanos, falta de equipamiento y que mediante la opinión de los habitantes, se pueda medir el grado de aceptación que se tiene por la vivienda, considerando entre estos, las condiciones de ubicación al estar alejados de los centros de trabajo, o del equipamiento social, educativo, de salud y cultural. El estudio ofrece información del fraccionamiento Senderos de San Isidro que se caracteriza por el abandono de casas y el incremento de la violencia urbana. También se 
localiza al límite de la mancha urbana, no habiendo ningún fraccionamiento o edificación colindante hacia el oriente, lo que en el horizonte visual, se define de exclusión o vacío. ${ }^{1}$

\section{Normatividad para la utilización de vacíos urbanos o espacios en desuso}

La normatividad para ocupar los vacíos urbanos es uno de los puntos medulares del presente escrito, vinculado al uso de zonas periurbanas, suscrito en el Plan de Desarrollo Urbano Sostenible de Ciudad Juárez, (PDUS) (IMIP, 2016: 19). Es importante enmarcar, que este plan identifica en el apartado de zonas diferenciadas la estrategia de densificación, el impulsar "el crecimiento hacia adentro y hacia arriba", teniéndola como una acción de prioridad para ocupar y promover el uso de los vacíos urbanos al interior de la ciudad, logrando así reducir las áreas subutilizadas. Otro propósito es utilizar los espacios en desuso, mediante el reciclaje de edificaciones, proyectos de uso mixto y construcción de vivienda vertical.

La justificación planteada por el PDUS (IMIP, 2016) para realizar una densificación es que los "indicadores de competitividad de instituciones oficiales nacionales y organismos internacionales", plantean que la ciudad compacta es donde se "vive mejor", justificando lo anterior menciona que se logra una mejor distribución de presupuesto y recursos municipales para equipamiento e infraestructura que en una ciudad extendida, ya que se logra una mejor cobertura y distribución de servicios a menor costo.

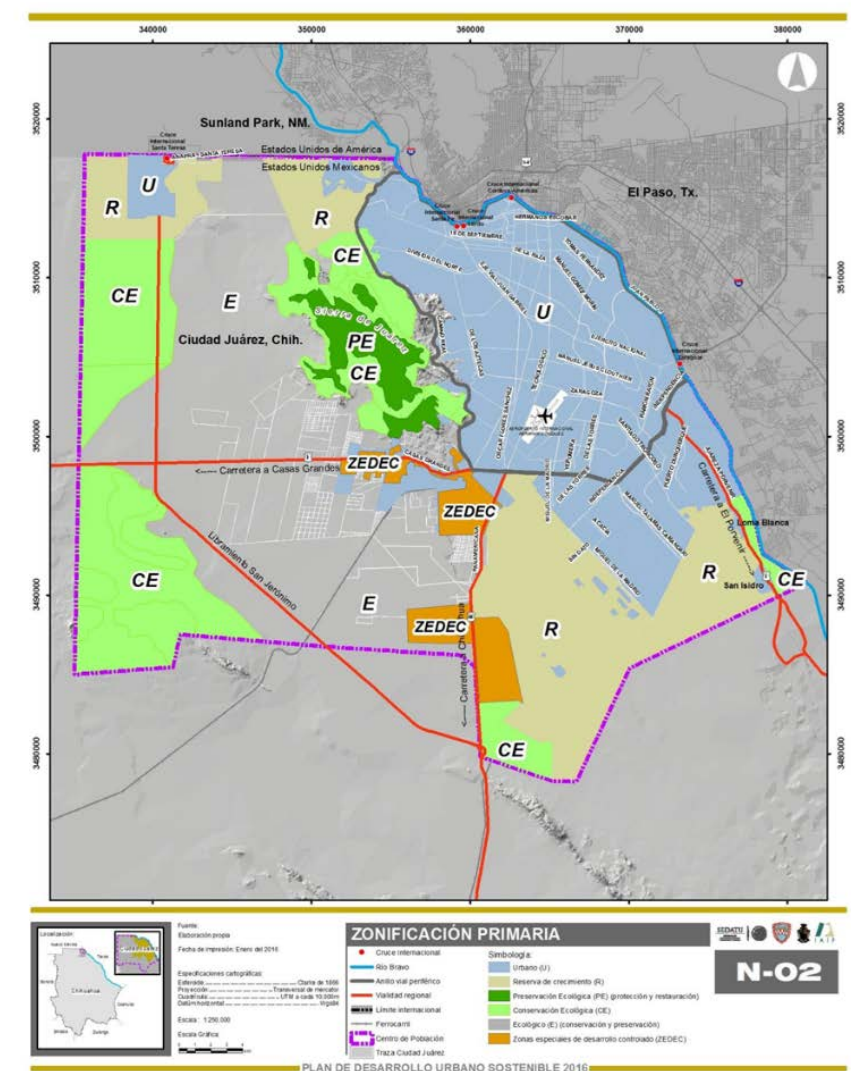

Figura 1. Traza urbana de Ciudad Juárez para ubicar Zona Urbana (U) y de Reserva $(R)$. (Fuente: Plan de Desarrollo Urbano Sostenible, normatividad (IMIP 2016: 16).

\footnotetext{
${ }^{1}$ Se considera el término exclusión cuando los habitantes están en lugares alejados, de desolación y que contribuyen a
} estar fuera de la ciudad, debido a la lejanía de los beneficios que ofrece. 
Es conveniente de puntualizar que en el Plan de Desarrollo Urbano Sostenible (IMIP, 2016), la zonificación para el desarrollo, establece restricciones, condicionantes y reglamentaciones dirigidas a la regulación de uso de suelo, según su ubicación, riesgo y extensión. Considera la división y clasificación de tres zonas que se deben abordar, según sus condiciones: a) Zona de Densificación Prioritaria; b) Zona Periurbana (dentro del área urbana identificada como "U", y c) Reservas de crecimiento "R" (ver figura 1). Estas reservas presentan condicionantes especiales para desarrollo a corto, mediano y larga plazo. El propósito es instrumentar y normar según las necesidades, considerando diferentes periodos de tiempo, con la finalidad de detener el crecimiento expansivo, de ciudad dispersa, desarticulada y donde se recorren grandes distancias, que disminuyen su competitividad.

Al potenciar las zonas o predios factibles de urbanizar, se deben considerar las normas vigentes de desarrollo urbano, entre las que se encuentran los aspectos de infraestructura, equipamiento, usos de suelo, vialidades, transporte, protección civil y medio ambiente. Lo anterior a partir de nuevas políticas federales, de los programas de organismos intersecretariales, que consideran las necesidades reales de la población, la ciudad, la legalidad y el apego a la política urbana actual" (IMIP, 2016).

Un segundo aspecto planteado en este apartado hace referencia a la normatividad para zonas periurbanas, donde de manera inicial, localiza el límite de las "zonas prioritarias de densificación" (IMIP, 2016), ubicándolas a una distancia entre 12 y $17 \mathrm{~km}$ del centro geográfico de la ciudad, según el PDUS (ver figura 2).

El crecimiento desarticulado tiene como problemática que la autorización de desarrollos habitacionales en las áreas de reserva o periurbanas, donde el PDUS hace su acotación para llevar a cabo los procedimientos de autorización, quien determina debe apegarse a la normatividad y legislación vigentes, realizando un estudio de factibilidad técnico que deben presentar los desarrolladores, con propuestas de acuerdo a la ley de Desarrollo Urbano Sostenible (DUS), el Reglamento de Desarrollo Urbano Sostenible (RDUS) y el mismo Plan de Desarrollo Urbano Sostenible (PDUS). 


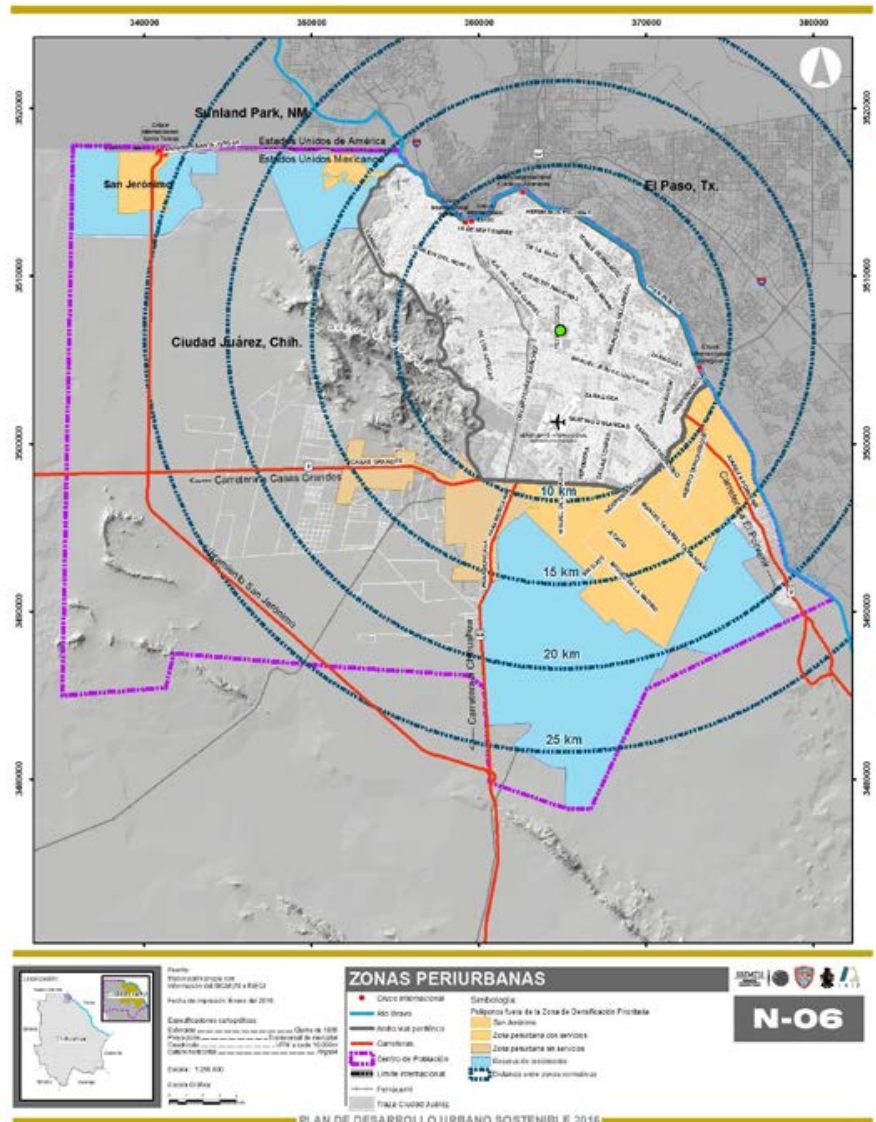

Figura 2. Distancias del centro geográfico donde se ubican las zonas periurbanas Fuente: IMIP Plan de Desarrollo Urbano Sostenible, normatividad (2016:49).

\section{Antecedentes: El proceso histórico del desarrollo.}

Ciudad Juárez ha abordado la planeación urbana a partir de Planes Reguladores desde 1958, en los cuales se abordaban de manera general la problemática de la ciudad; ese año el plan tenía como peculiaridad de la época, resaltar las dinámicas comerciales, de turismo y la vida nocturna característica. La dispersión por ubicación de construcciones localizadas al sur y suroriente del centro geográfico, era motivo de preocupación desde esa época, ya que las distancias a recorrer eran considerables (IMIP, 2016).

El plan regulador de 1962, proponía "dar respuesta a problemáticas de las ciudades fronterizas" a través de una zonificación coherente de los usos de suelo, teniendo como propósito fundamental, la creación de un organismo que vigilara los instrumentos de regulación y planeación existentes (IMIP, 2016 :5).

En 1979 el Plan de Desarrollo establece otros objetivos, generar una trama urbana que conectara de manera eficiente las diferentes zonas económicas de la ciudad, ya que las diferencias eran muy marcadas, al poniente se ubicaba la población de ingresos más bajos, mientras que al norte la de recursos económicos solventes (IMIP, 2016).

En el plan de 1984 se pretendía básicamente regular el crecimiento de la ciudad, que tuviera en consideración el respeto de las áreas naturales, la topografía y drenes existentes, con el propósito de mantener y controlar los recorridos de las aguas pluviales, al tratar de impedir un desarrollo desarticulado, con zonas desvinculadas 
y aisladas, de los sectores más vulnerables y que elevaran la calidad de vida por medio de la viviendas, sin embargo, esta década fue la de menor producción de casas (IMIP, 2016).

En 1989 el Plan de Desarrollo Urbano, propone una reorganización de la estructura urbana, donde la industria tenía un papel preponderante, mediante una distribución equilibrada de esta actividad, y el propósito de aprovechar al máximo la infraestructura existente, considerando aumentar la densidad de la población de 74 a 76 habitantes por hectárea.

El Plan de Desarrollo de 1995 proponía el objetivo de elevar el nivel de vida de los habitantes, con un ordenamiento programado y racional, que reconocía que a pesar de lo propuesto y actualización de planes no se había logrado (IMIP, 2016).

La actualización del 2003, hace referencia a una ciudad con calidad de vida y sustentable, al contar infraestructura, equipamiento y seguridad publica eficiente, mediante el uso razonable de recursos, de una ciudad incluyente, con buena educación, que fortalezcan las actividades y se diversifique la economía a través de una administración efectiva, transparente y eficiente por parte de las autoridades (IMIP, 2009).

El Plan del 2009, también hace referencia a la calidad de vida de los habitantes, se proponían directrices y lineamientos para un "adecuado ordenamiento territorial". Al igual que en el plan 2003, se abordó el mantenimiento del equipamiento y preservación de los elementos naturales, que lograra un crecimiento ordenado y armónico de la ciudad (IMIP, 2009).

Los Planes Parciales, fueron estrategias para justificar la integración del territorio al área urbana, teniendo diferentes épocas, propósitos y normas para la reserva del crecimiento; algunos de los autorizados en Ciudad Juárez son: el Periférico Camino Real (3,971.72 ha.), El Barreal y Oriente San Isidro (2,836.26 ha.), Oriente XXI primera etapa (1596.91 ha.) y San Jerónimo (3,502.73 ha.), los cuales actualmente cuentan con zonificación secundaria y usos de suelo para su ocupación a mediano plazo (IMIP, 2016).

En 2014-2015 se autorizan tres áreas de reserva territorial adicionales: Plan Parcial "Independencia 21" (520 ha.), Plan Parcial "Promotora del Norte" (212.56 ha.) y Plan Maestro "Cumbre de Andares" (71.00 ha.), teniendo como condición para su aprobación el uso industrial exclusivamente, quedando prohibido el uso habitacional (IMIP, 2016).

\section{La habitabilidad factor determinante para el crecimiento urbano}

Ciudad Juárez por ser frontera es un punto de atracción para población migrante que llega con la intención de cruzar hacia los Estados Unidos de Norteamérica en busca de mejorar sus posibilidades económicas; sin embargo, gran parte no logra este propósito y se asienta temporalmente; termina laborando en la industria maquiladora que ofrece beneficios sociales y acceso a servicios urbanos y vivienda, lo que define su residencia permanente.

Lo anterior genera el crecimiento de la ciudad, la necesidad de alojamiento conduce a la necesidad de nuevos fraccionamientos para dotar de vivienda a los nuevos habitantes, sin embargo, la distribución territorial que se genera por parte de los desarrolladores y la autorización de planes reguladores de crecimiento produce otros conflictos, la creación de parques industriales en esas zonas alejadas del centro geográfico atrae el desarrollo de conjuntos habitacionales en teoría para que el centro de trabajo quede cerca de la zona habitacional, sin embargo, estas soluciones o planeaciones están dejando grandes espacios vacíos dentro de los limites urbanos debido a un proceso de especulación dentro del mercado inmobiliario. 
Bazant menciona que las ciudades mexicanas tienen "crecimiento explosivo y anárquico" (2011:28), donde el desarrollo se va dando en condición de conveniencias políticas y personales, sin tomar en cuenta los planes reguladores de desarrollo, que plantean o proponen desarrollos eficientes, con usos eficientes de espacio, infraestructura y equipamiento.

Cuando los planes de desarrollo o estratégicos, hablan de calidad de vida, se hace referencia directa a la habitabilidad urbana, donde los aspectos medioambientales, el planteamiento morfológico y la calidad del equipamiento e infraestructura son vitales para ofrecer condiciones de progreso en una colonia.

La habitabilidad se impacta, cuando las ubicaciones geográficas, de los desarrollos están alejados del centro de la ciudad. En este aspecto hace referencia a las distancias que hay que recorrer para traslados, principalmente a lugares de trabajo y escuelas, donde el sector inmobiliario, el uso y el valor no se relacionan con su localización. Pedro Abramo menciona que debe existir un beneficio adicional cuando se tienen largas distancias respecto la ciudad, es decir que "para que el individuo continúe teniendo un nivel de utilidad de la combinación distancia- espacio idéntico al nivel anterior, el distanciamiento deberá ser compensado por un aumento del consumo de espacio" (2010: 30).

No se puede dejar fuera el tema de equipamiento, ya que no todo se encuentra en áreas periurbanas, por lo que algunos habitantes tienen que trasladarse a otros sectores de la ciudad, ya sea para recibir atención en el sector salud o realizar trámites de índole púbico o privado.

En este sentido Alcalá (2007), citado por Moreno (2008), hace referencia a la relación entre la vivienda y su entorno, así como la integración física con la ciudad, de tal forma que exista una buena accesibilidad a servicios, transporte, equipamiento y espacios públicos que forman parte de la estructura urbana, esto plantea que debe haber una correlación adecuada y accesible entre todos los espacios o áreas de la ciudad.

\section{Metodología}

El presente artículo surge del análisis de habitabilidad urbana realizado en varios fraccionamientos de Ciudad Juárez. El proceso metodológico para llevar a cabo la investigación se dio en varias fases:

1) La primera se centró en un acercamiento bibliográfico al concepto de habitabilidad y una revisión a las normativas vigentes que permitieran realizar cuestionamientos a las propuestas, el propósito fue identificar comparativas de lo textual a lo real.

2) En la siguiente etapa se revisó la traza urbana con el propósito de identificar vacíos urbanos o predios en desuso, para entender la dinámica de crear de nuevos desarrollos habitacionales.

3) El tercer paso fue caracterizar los fraccionamientos al seleccionar los adecuados para hacer el estudio buscando que cumplieran con características previamente definidas, entre las que se encuentran: localización -estar alejada de la zona centro; tener vivienda mínima con una construcción de alrededor de 40.00 metros cuadrados; predios de 120.00 metros cuadrados; antigüedad de alrededor de los 10 años de fundada. Además, se evaluaron factores de percepción como son: apreciación de seguridad y de vigilancia policial, aceptación del entorno urbano (parques y limpieza de calles) y arraigo al sector de la vivienda.

4) La cuarta etapa consistió en la aplicación de cuestionarios, teniendo una muestra de 54 , la cual se determinó en condición del número de viviendas habitadas según el censo de población y vivienda del Instituto Nacional de Estadística y Geografía (INEGI, 2015).

En la selección del fraccionamiento, fue importante que se pudiera acceder al sector de forma segura para la aplicación de encuestas, en este caso solo se presentan resultados de uno de los casos de estudio. 
En la definición de la muestra se consideró un 40 por ciento de viviendas abandonadas lo que posibilitó aplicar el número de cuestionarios que nos dieran una factibilidad de error de menos del $5 \%$, estando dentro de los parámetros establecidos por estándares de calidad.

La metodología es de tipo mixto ya que se utilizan gráficas y valores cuantitativos de los resultados de encuestas, así como la corroboración perceptual con base a la opinión de las personas. Es importante considerar aspectos de valoración subjetiva que se relacionan con el logro de contar con una vivienda propia, y el interés de mejora para su sector.

\section{Zona de estudio}

El desarrollo habitacional de Senderos de San Isidro, se ubica al sur oriente de la ciudad, a una distancia en línea recta de aproximadamente 25 kilómetros del centro. Es un fraccionamiento con una extensión aproximada de $744,240.30$ metros cuadrados y cuenta con 2731 viviendas con lotes promedio de 120.00 metros cuadrados (figura 3).

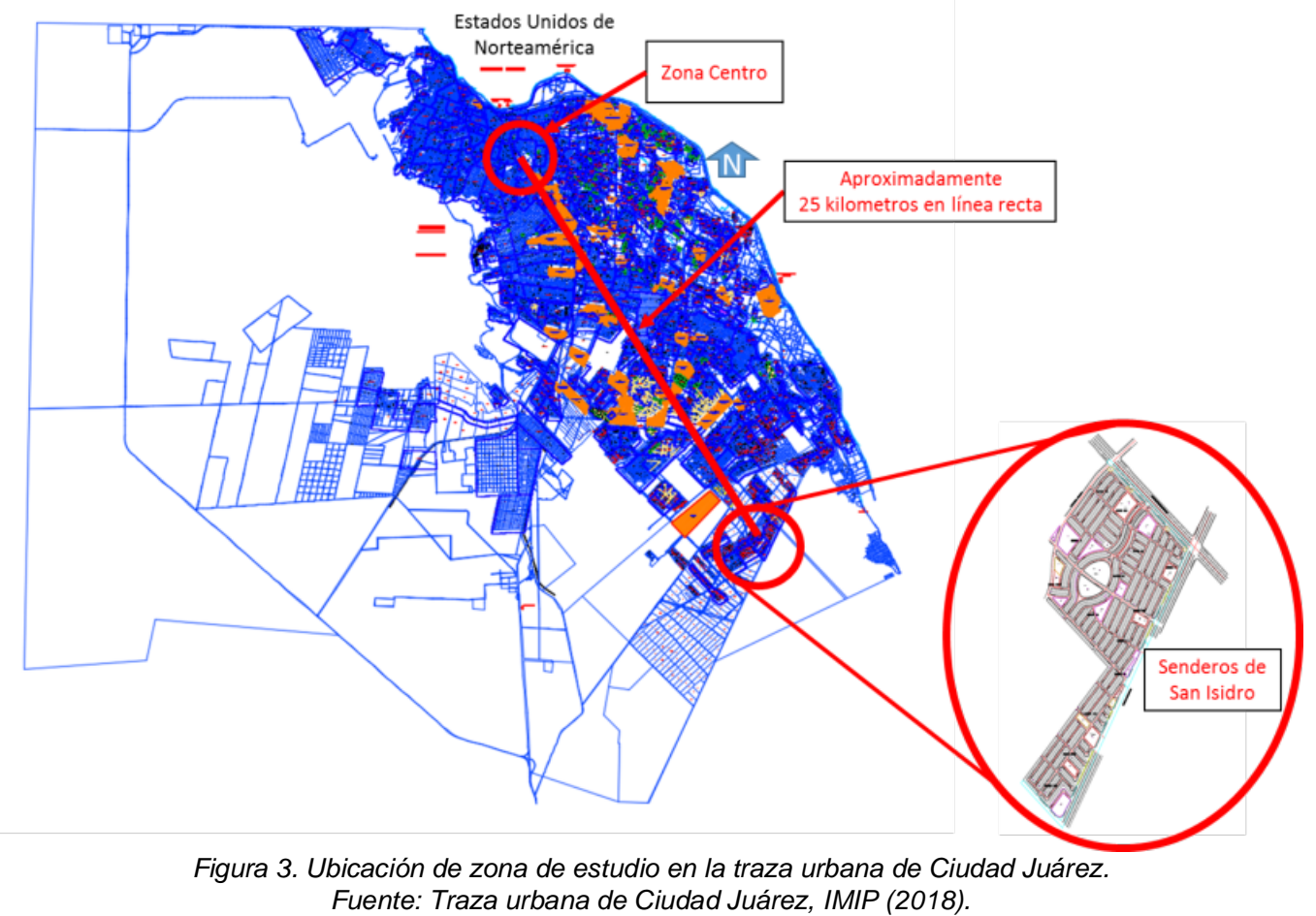

\section{Resultados}

Sobre los resultados obtenidos de la aplicación de encuestas y observación de campo, se identifican algunos aspectos relacionados con la habitabilidad urbana, teniendo en cuenta factores de localización, de percepción y valoración de ambientes conforme a estándares normativos, que sirven de parámetros para explicar situaciones de aceptación o rechazo de su sector o propiedad. 
En cuanto a la localización de la vivienda se tiene lo siguiente: Los habitantes en general utilizan como medio de transporte para trasladarse al trabajo el autobús de personal que proveen las empresas, o en su defecto el transporte público de la ciudad. Pocos habitantes de trasladan a pie y un grupo mínimo lo hace en automóvil propio. Según los resultados, el 38.9 por ciento de los encuestados utiliza el transporte de personal para ir a la fábrica; otro $25.9 \%$ lo hace en transporte público. Es importante acotar que la industria maquiladora, es la que provee transporte gratuito para sus empleados. El 9.3\% se moviliza en automóvil y el 3.7\% lo hace caminando, debido a que son empleados cerca del área habitacional, ya sea en comercios o negocios propios. (ver figura 4).

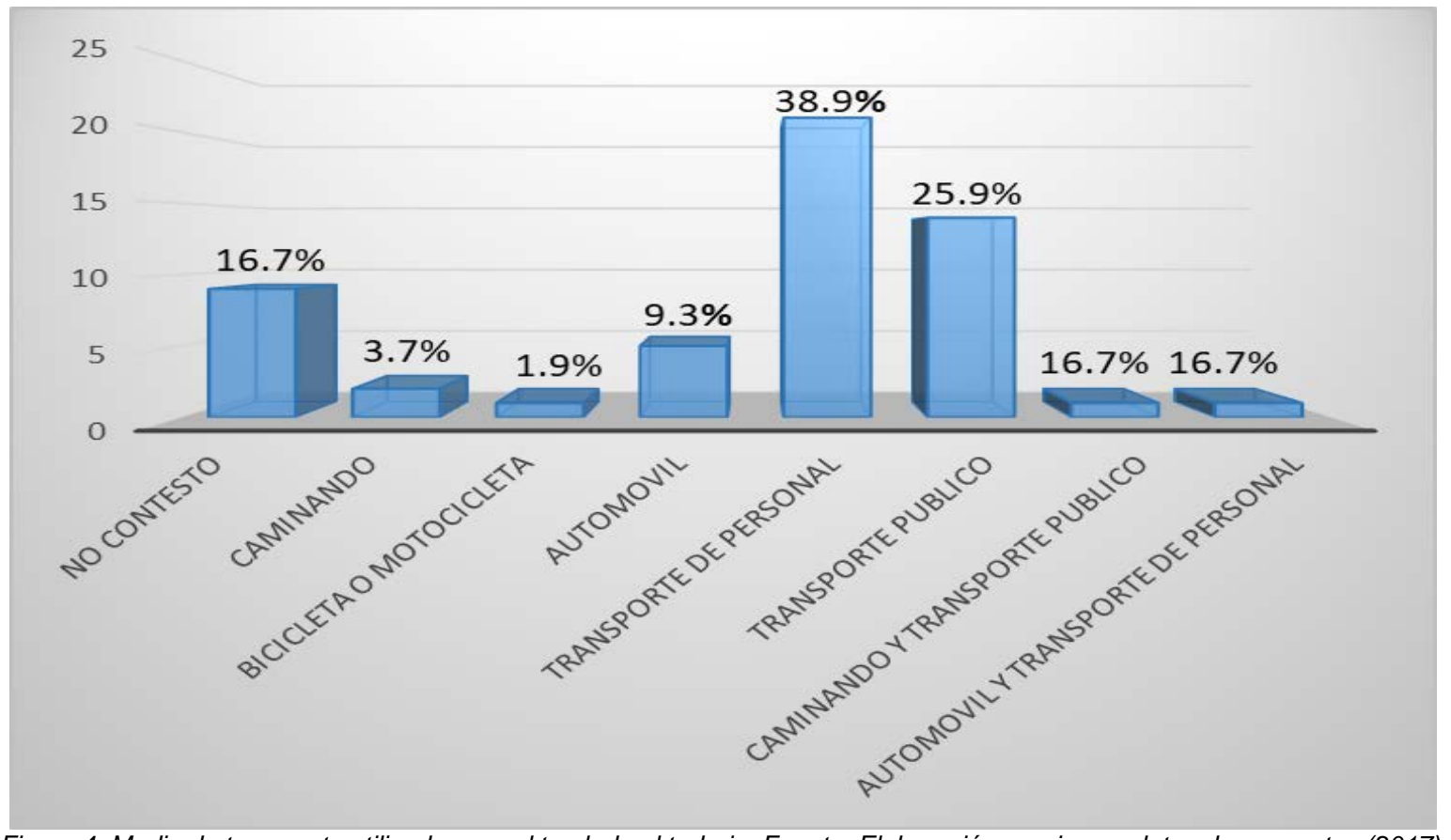

Figura 4. Medio de transporte utilizado para el traslado al trabajo. Fuente: Elaboración propia con datos de encuestas (2017).

El factor de localización en estos fraccionamientos y el tipo de empleo implica que los servicios de transportación estén bien definidos desde que los residentes se fueron a habitar el fraccionamiento, ya que los puntos de ubicación de paradas del transporte público y llegada de camión de personal, se ubiquen cercanos al domicilio. En este caso el 57.4 por ciento menciona que se traslada caminando más de 6 cuadras (600 metros) para abordar el transporte; el 20.4 por ciento lo toma entre 3 a 5 cuadras, en ambos casos esto implica entre 5 y 10 minutos de tiempo para llegar al lugar (ver figura 5). 


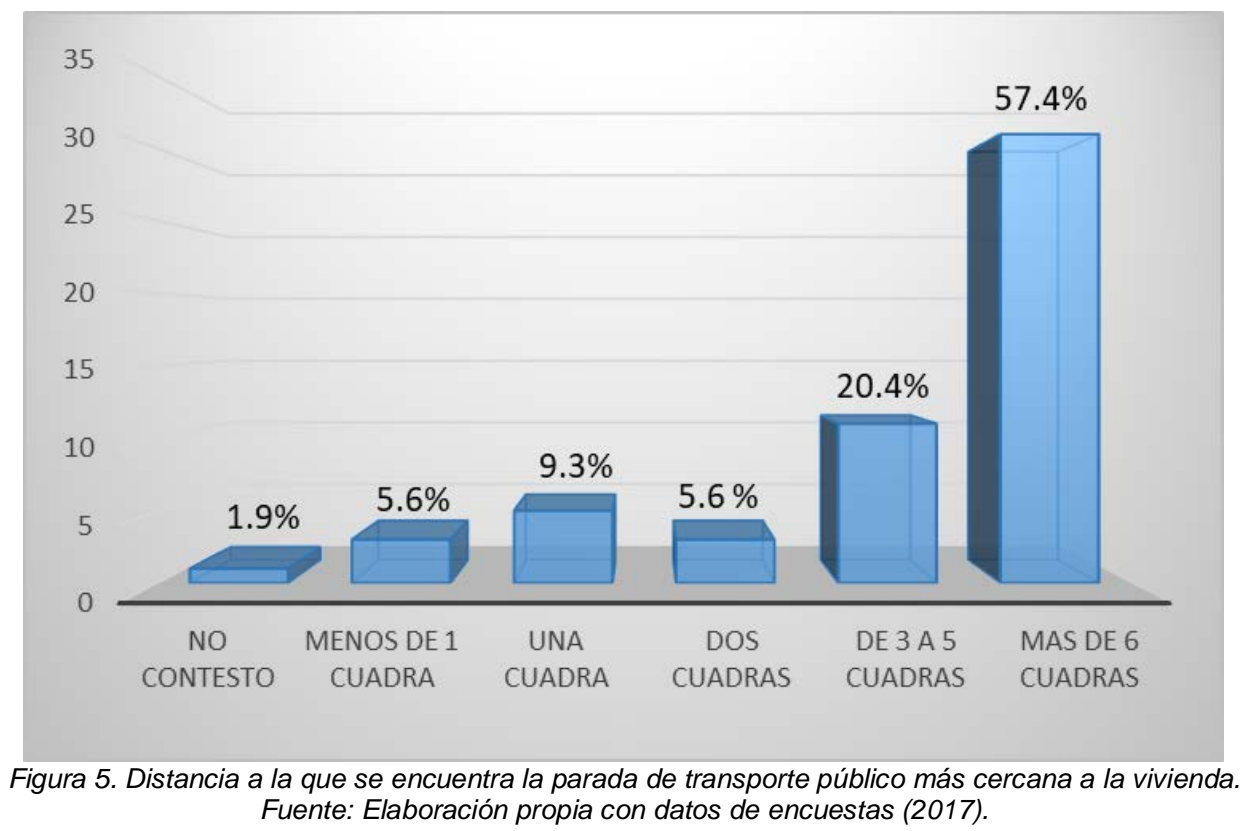

En el caso del tiempo de traslado puede considerarse que los trabajadores de maquila son los que se localizan más alejados de su sitio laboral, ya que el 38.9 por ciento (mismo que usa transporte de personal) dura entre 30 a 60 minutos para arribar al lugar de trabajo. Los empleados que usan transporte público, invierten de 1 a 2 horas; y el 20.4 por ciento llega en menos de media hora, ya sea porque va en auto propio o caminando al estar en su sector (figura 6).

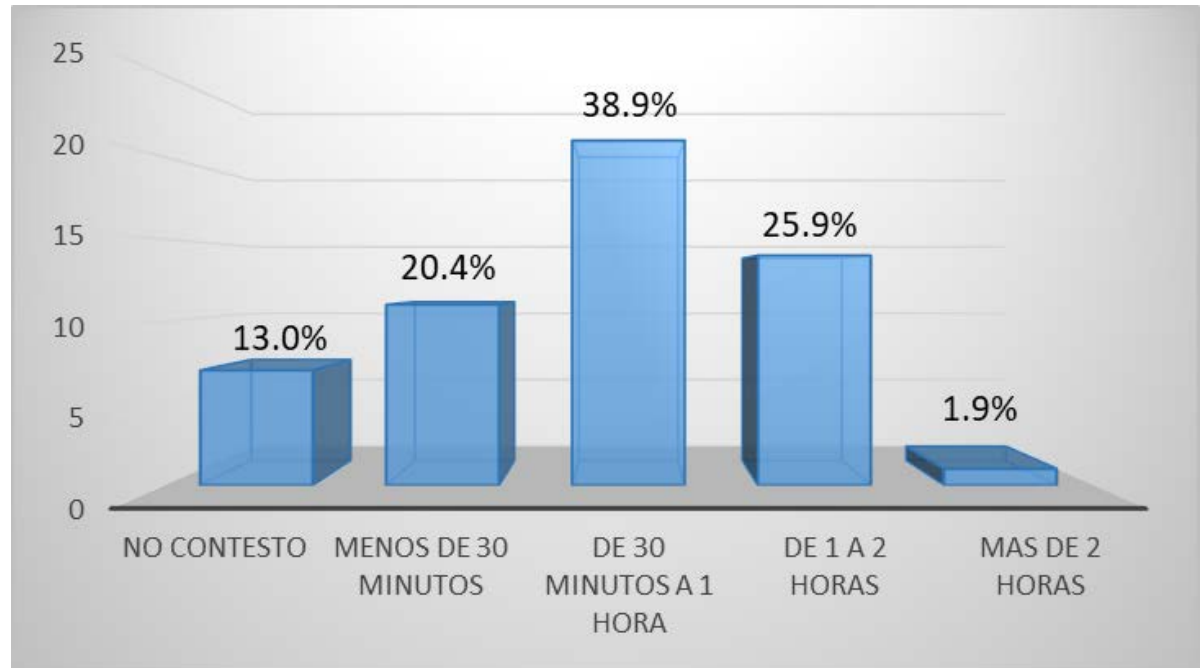

Figura 6. Tiempo utilizado para trasladarse al trabajo (Fuente: Elaboración propia con datos de campo, 2017).

Las distancias y recorridos para llegar a las escuelas también se consideraron en la encuesta y se encontró que un 53.7 por ciento se van caminando; el 16.7 por ciento se traslada en transporte público y solo el 7.4 por ciento en automóvil (ver figura 7). En condición de lo anterior se identificó que los tiempos de traslado son de menos de 30 minutos para el 70.4 por ciento de los encuestados. 


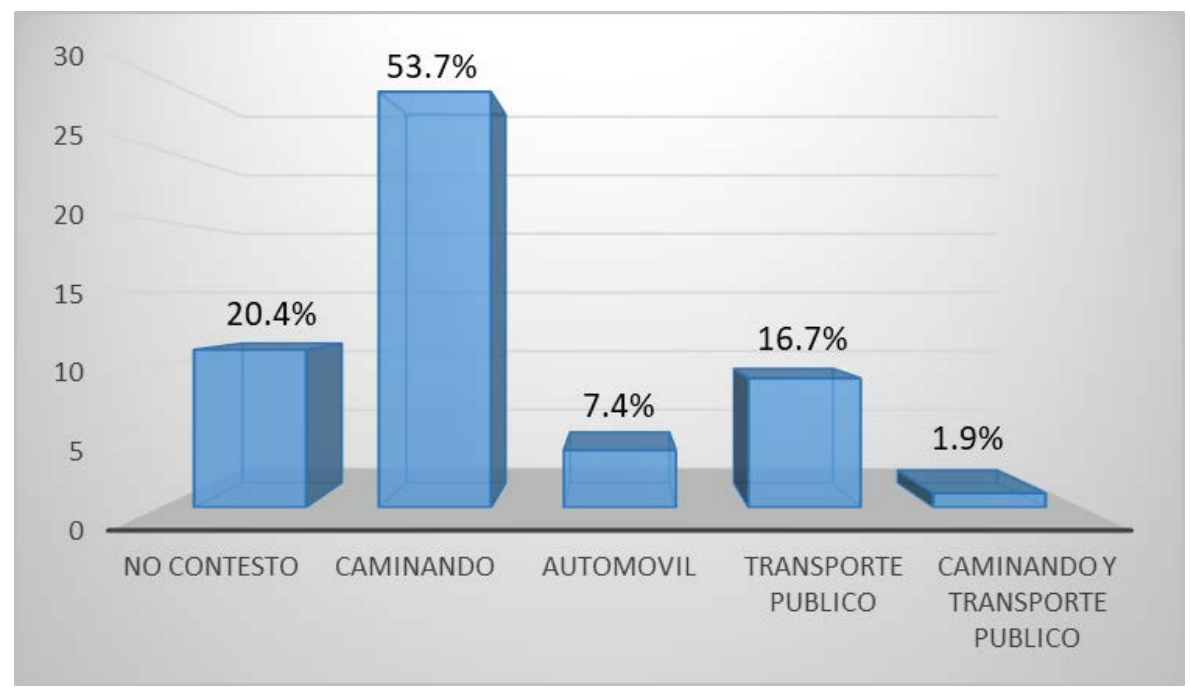

Figura 7. : Medio de transporte utilizado para trasladarse a las escuelas (Fuente: Elaboración propia con datos de campo, 2017).

En cuanto al equipamiento de salud, en los fraccionamientos periurbanos en cuestión de distancias, se carece de oportunidades de subsanar alguna emergencia, ya que el 48.1 por ciento de los encuetados del fraccionamiento, tardan entre 30 y 60 minutos para llegar al servicio médico más cercano; un 33.3 por ciento tarda en llegar de 1 a 2 horas (figura 8). En ambos casos la lejanía de los beneficios de salud a los que tienen derecho los trabajadores del sector formal se ven restringidos por la distancia y tiempo de traslado.

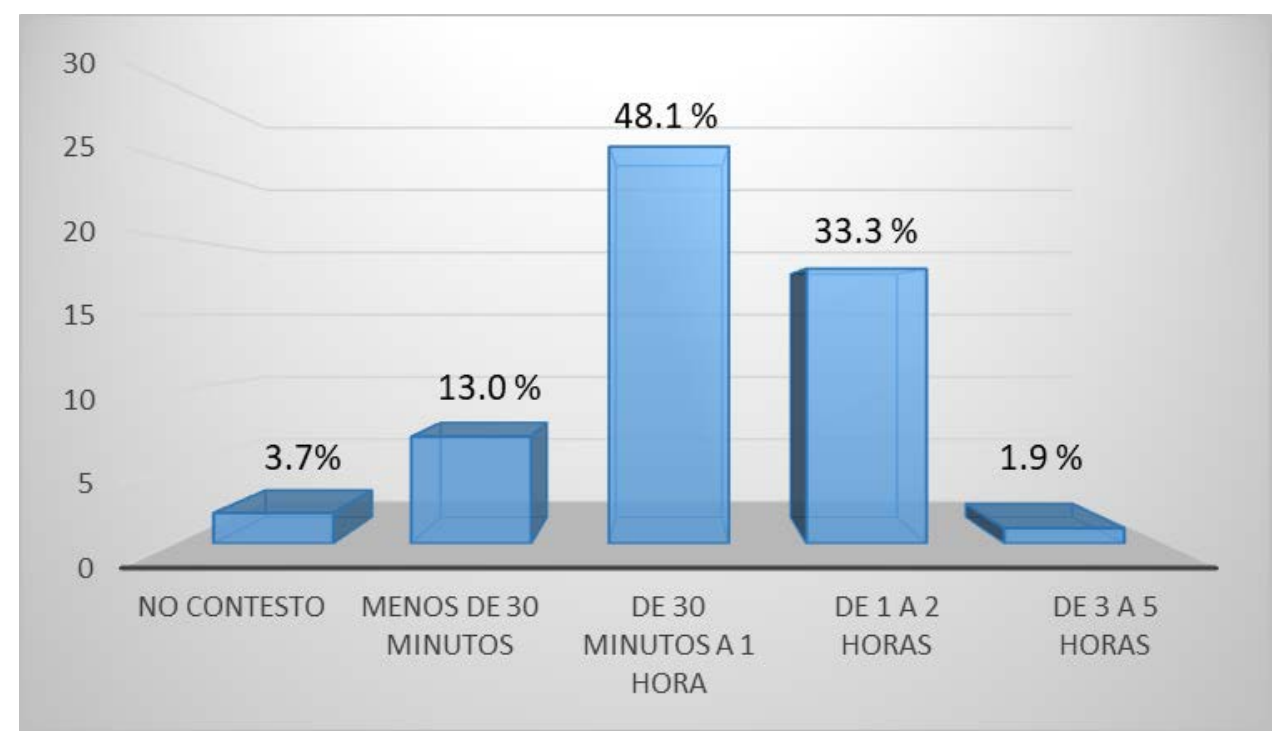

Figura 8. Tiempo utilizado para trasladarse a un centro de salud. Fuente: Elaboración propia con datos de encuestas (2017).

La calidad del espacio público que se ofrece por medio de parques y áreas verdes, para los encuestados dista mucho ser el lugar que les ofrecieron al venderles la casa, ya que el 79.6 por ciento lo considera de mala 
calidad; el 14.8 por ciento lo evalúa regular, y solo el 3.7 por ciento le da un resultado bueno, condición que implica que existen pocas opciones que revaloricen la propiedad den ese (figura 9).

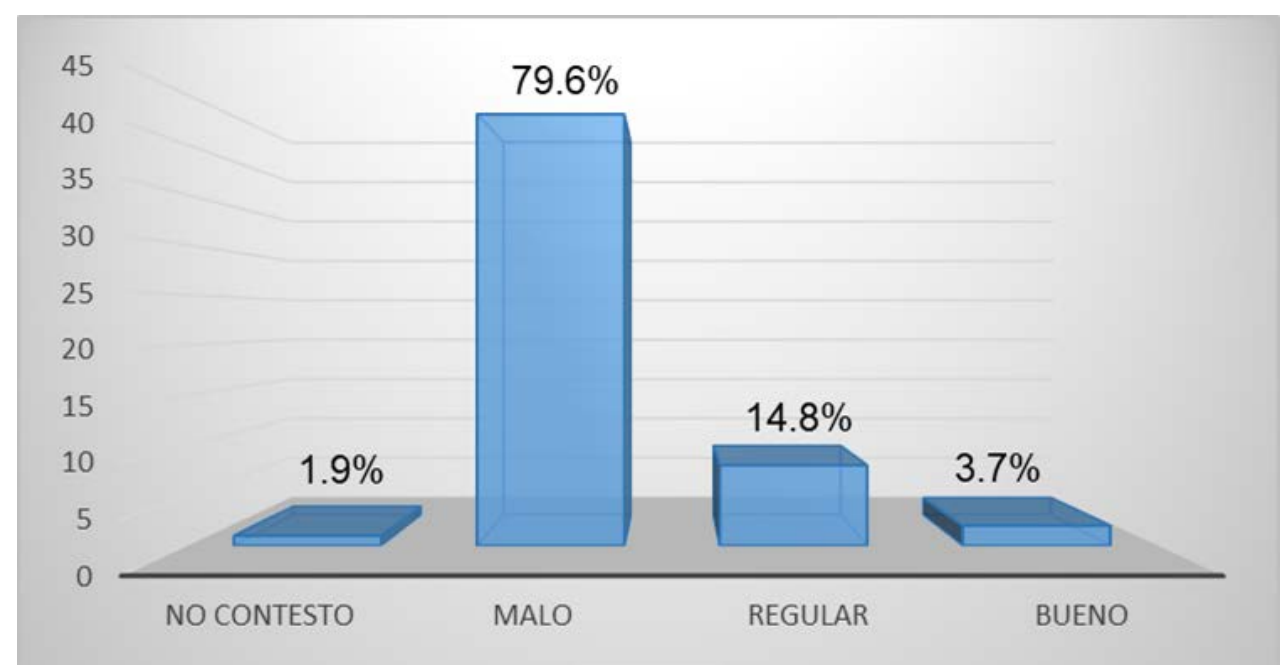

Figura 9: Evaluación de las áreas verdes de su colonia. Fuente: Elaboración propia con datos de encuestas (2017).

El espacio público de calles y banquetas se distingue por el deterioro permanente y de mala calidad, ya que son ambientes poco limpios y en mal estado, según lo evalúan el 75.9 por ciento de los encuestados, el 14.8 por ciento le valora regular y el 7.4 por ciento bien. Las opciones que tienen los habitantes para contar con calles y banquetas limpias, se fundamentan en buenos hábitos de limpiar y mantener aseados el frente de la casa, por medio de la acción de los propios residentes, únicamente así se les puede proveer de un lugar en condiciones de habitabilidad urbana (ver figura 10).

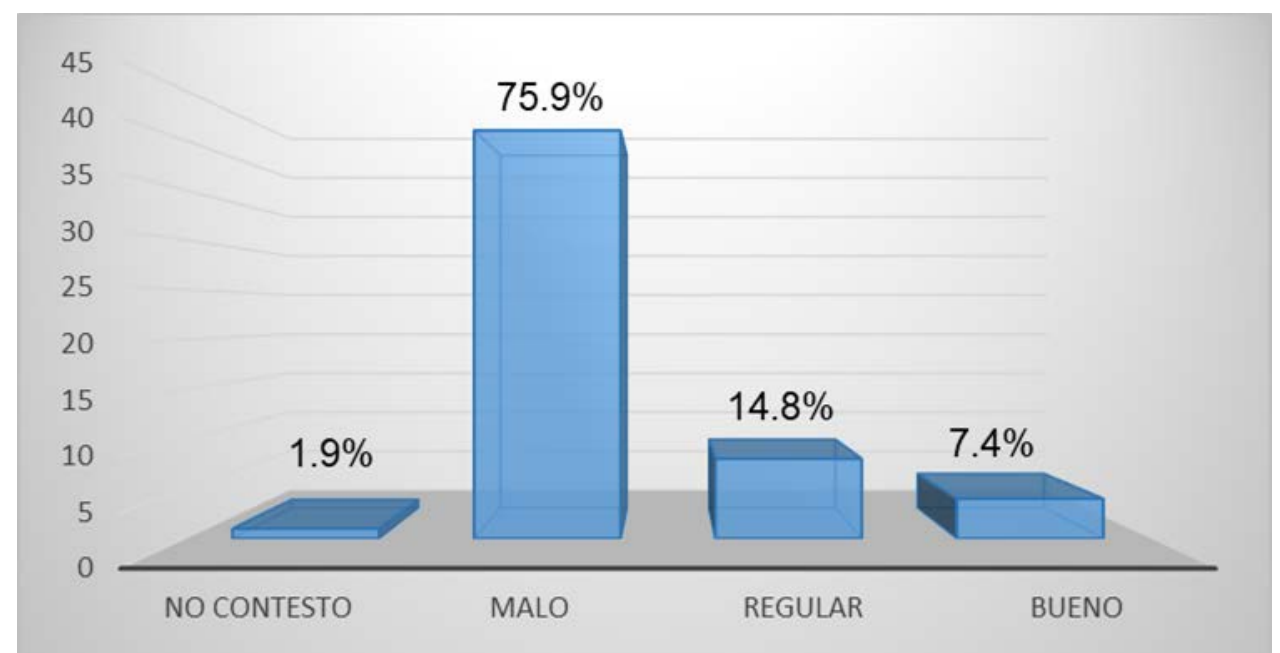

Figura 10: Evaluación de la limpieza de calles en su colonia. Fuente: Elaboración propia con datos de encuestas (2017).

En el análisis de habitabilidad urbana la seguridad del sector se considera como un factor preponderante para el agrado de los habitantes por su colonia, en este aspecto el 48.1por ciento, considera que es regular, un 20.4 por ciento dice que es inseguro contrario al 16.7 por ciento que lo considera seguro. Cabe destacar que un 
11.1\% lo considera muy inseguro, esto con referencia a que el incremento de sucesos delictivos dirigidos a las personas es frecuente y se carece de alguna estrategia para mitigarlo (ver figura 11).

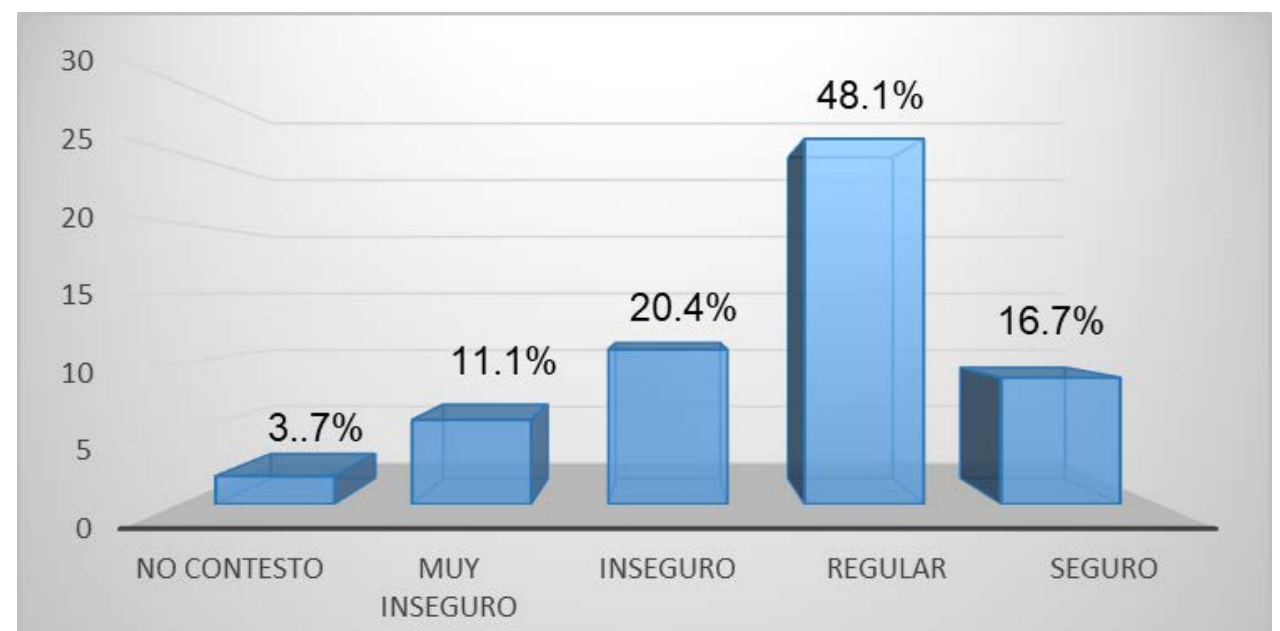

Figura 11. Percepción del ambiente de seguridad en su colonia. Fuente: Elaboración propia con datos de encuestas (2017).

Al preguntarle a los encuestados como consideran el servicio de seguridad pública municipal que se les brinda por medio de las instancias gubernamentales, aproximadamente el 63 por ciento lo consideró malo, solo un poco más del 29 por ciento lo percibe regular y el 7.4 por ciento lo considera bueno. Las razones principales que mencionaron para tener esa percepción fue la falta de patrullaje que, en algunos casos consideraron es mínima, con pocas veces a la semana o solo se presentan posterior a un evento delictivo, para hacer rondín por el sector (ver figura 12).

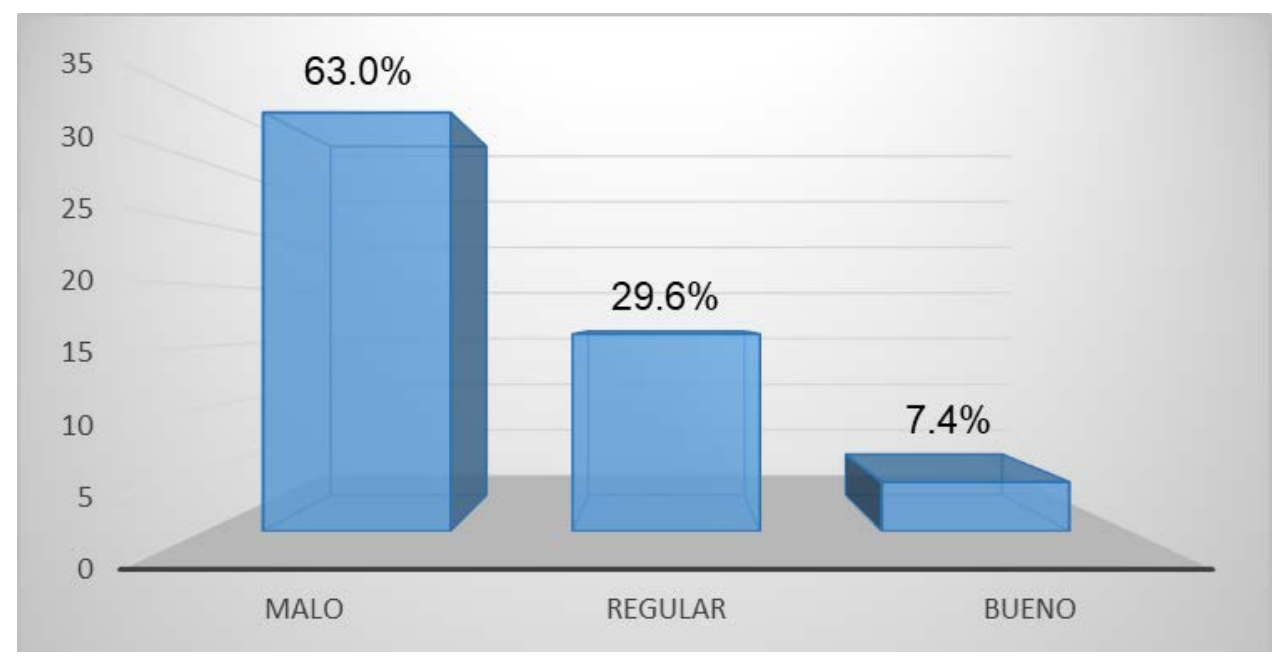

Figura 12: Evaluación del servicio de seguridad pública en su colonia. Fuente: Elaboración propia con datos de campo, (2017).

Un mecanismo de valoración para desarrollar programas de participación y colaboración de los habitantes coadyuvando en las mejoras del lugar, es plantearles la opción de permanencia o no en su fraccionamiento, teniendo los mismos beneficios de financiamiento con que cuentan en ese momento. En respuesta se tuvo que el 51.9 por ciento esta dispuesto a cambiarse ya sea, a una colonia cerca de la familia, a un fraccionamiento 
más cercano, a uno aledaño al centro y a otra ciudad. ${ }^{2}$ El 49.1 por ciento permanecería con su propiedad actual (Ver figura 13).

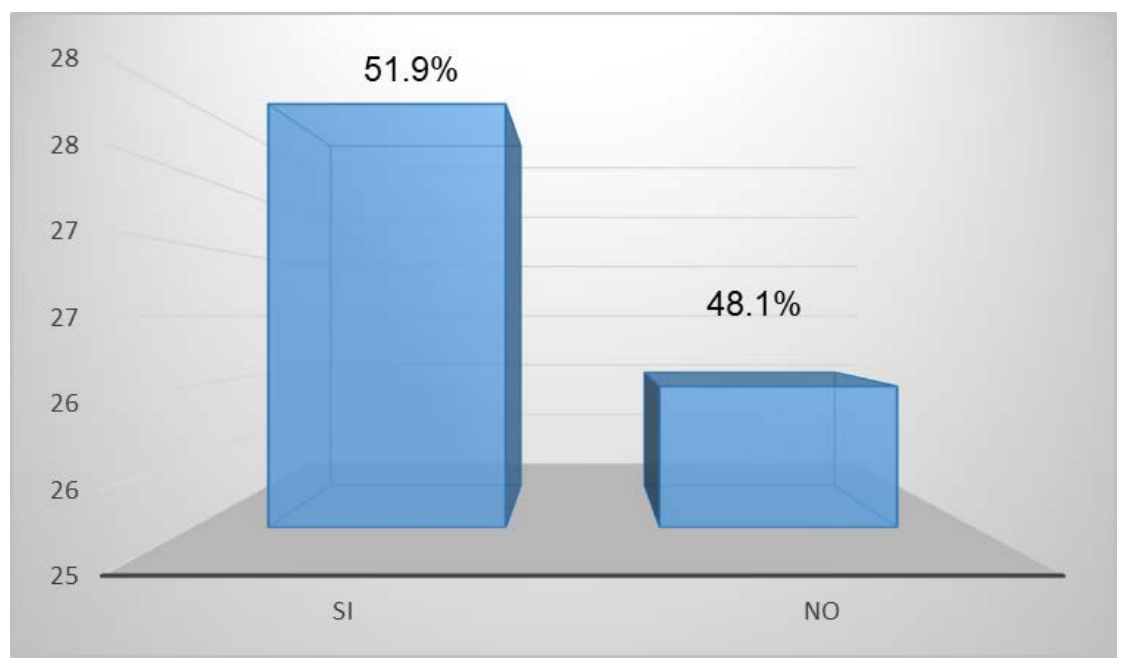

Figura 13: Opinión sobre cambiarse a otra zona. Fuente: Elaboración propia con datos de encuestas (2017).

En las condiciones de arraigo se valora las posibilidades de movilidad en la ciudad o a otra localidad. En este caso el 59.1 por ciento considera moverse, pero solo el 11.1 por ciento lo haría cerca del centro y otro 11.1 por ciento cerca de la familia, situación que beneficia la generación de redes de apoyo mutuo. El 20.4 por ciento busca irse más cerca del centro, lo que facilitaría su acceso a las ventajas que ofrece la ciudad y el 9.3 por ciento se movería a otra ciudad, lo que impactaría en el retorno de migrantes a su lugar de origen (ver figura 14).

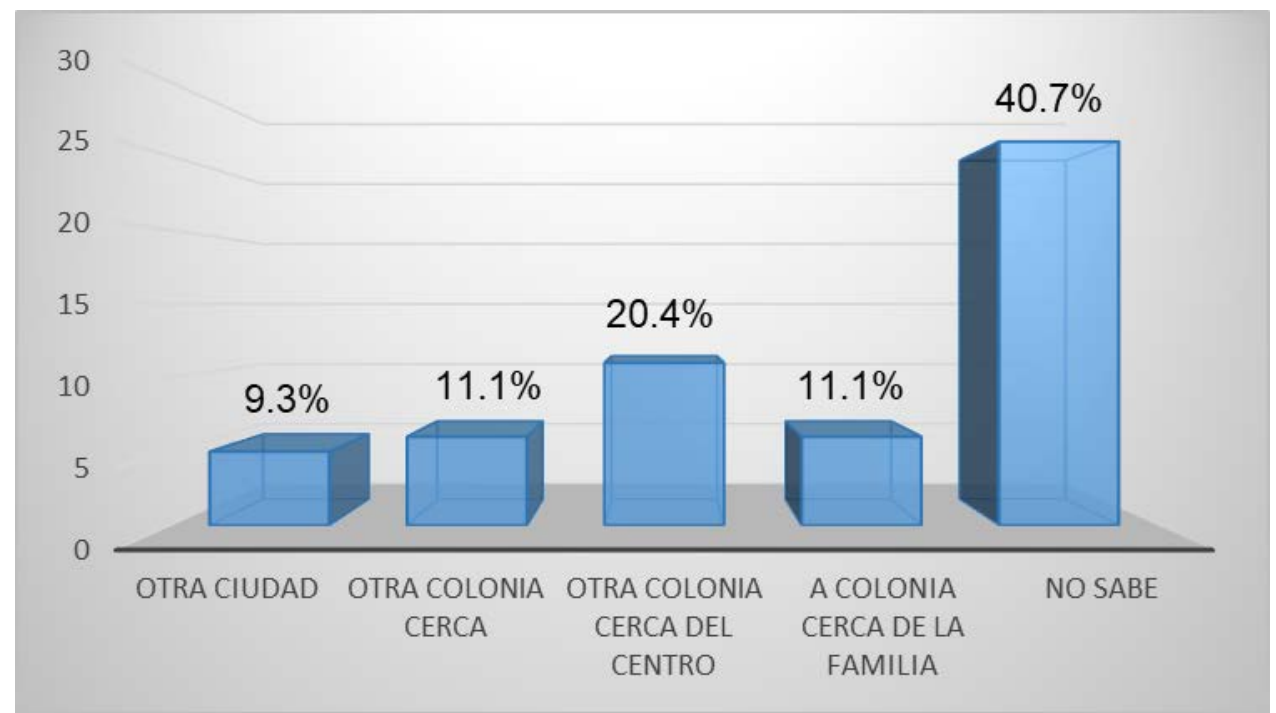

Figura 14: Sitio al que prefiere cambiarse. Fuente: Elaboración propia con datos de encuestas (2017).

22 En 2012 el Gobernador de Veracruz promovió el traslado masivo de habitantes originarios de ese Estado para alejarlos del ambiente inseguro que prevalecía en la ciudad, este hecho en este momento no tendría el mismo efecto, ya que sólo el 9.3 por ciento se movería a otra localidad. 
En cuanto a la opinión de orgullo o preferencia de vivir en el fraccionamiento, el 51.9 por ciento respondió que porque es su casa -orgullo de ser propietario-, mientras que el $14.8 \%$ percibe que el lugar es tranquilo, el $5.6 \%$ considera que le gusta en general el fraccionamiento y un $13 \%$ no le gusta nada del fraccionamiento, ya sea por ser un sitio inseguro, descuidado y con problemas de calidad del entorno (ver figura 15).

\section{Conclusiones}

Los planes de desarrollo hacen énfasis en la calidad de vida que debe existir para garantizar a las familias de trabajadores el acceso a una vivienda digna, según el artículo 4to. Constitucional sobre garantías individuales en México. Sin embargo, se autorizan zonas alejadas con opciones poco factibles para que se pueda proveer ambientes de calidad a partir de una ciudad planeada que tiene como propósito mejorar la calidad de vida de estos residentes. Las estrategias e instrumentos de regulación para la ubicación del equipamiento se disponen dentro del proyecto urbano, pero en realidad no se cuenta con estos espacios, están solos, baldíos, en condiciones de deterioro o vandalizados, en detrimento de incidir negativamente en la habitabilidad urbana.

Los vacíos urbanos y predios en desuso, dentro de la ciudad, se definen en los planes de desarrollo como prioritarios para su urbanización donde la normatividad está regulada para su cumplimiento, en la realidad, el 32 por ciento de la extensión territorial de la ciudad está definida por predios en estas condiciones.

El tiempo que utilizan las personas para llegar al trabajo, hace evidente que estas zonas habitacionales no se ubican cercanas al empleo, ya que los residentes tienen que ocupar de media hora hasta 2 horas de traslado, lo que hace ineficiente cualquier movilización en transporte público o de personal, en cuanto a consumo energético, de tiempo extra y de generación de contaminación. El uso de transporte de automóvil o camión de personal o público por más de media hora tiene impacto en la calidad de vida de los trabajadores quienes destinan entre de un 12.5 al 50 por ciento de tiempo adicional a sus 8 horas de trabajo, los que disminuye sus opciones de descanso y convivencia con la familia. Se infiere que las distancias no pueden ser recorridas caminando ya que la movilidad depende del transporte y/ o automóvil, teniendo que la creación de los nuevos parques industriales en ese sector no forzosamente son la fuente de trabajo de los habitantes de estos fraccionamientos.

Las distancias de traslado a las instituciones de educación son cortas, las personas se movilizan a estas caminando; aspecto que además de contar con los resultados de las encuestas, se pudo constatar por observación de campo donde grupos de estudiantes se trasladaban a las escuelas del sector. Se infiere que el equipamiento educativo a nivel preescolar, básico y secundaria está cubierta en el sector. Se carece de escuelas de nivel preparatoria o medio superior cercanas, lo que afecta la continuidad de los estudios de jóvenes en la mayoría de los fraccionamientos periurbanos. Esta escolaridad se ve trunca debido a que menos del 40 por ciento puede acceder al bachillerato, ya que la matrícula de egreso de la secundaria, no puede ser absorbida, ya que no se tienen espacios suficientes, ni cercanos a esta zona. Esto evidencia que por la edad de expulsión de los jóvenes se condiciona para que sean fácilmente atrapados por los centros laborales cercanos.

En cuanto a los servicios de salud, a pesar de que más del 60 por ciento cuenta con beneficios de atención médica, porque se emplean en trabajos formales, las posibilidades de acceder en una emergencia son casi nulas ya que destinan de 1 a 2 horas para llegar al hospital. La zonificación de este equipamiento dentro de la ciudad se concentra en la zona nororiente, a más de 20 kilómetros de los hogares en estudio. Además, los negocios identificados en el sector tampoco priorizan el servicio de atención médica particular, lo que establece 
condiciones de segregación y exclusión de los habitantes y les coloca en condiciones de vulnerabilidad en momentos de contingencia sanitaria.

El derecho a la ciudad implica contar con espacios de sano esparcimiento definidos en la normatividad como áreas verdes y recreativas, teniendo que aproximadamente el 75 por ciento de los encuestados considera que estos espacios están en malas condiciones, son insuficientes y carecen del equipamiento urbano y vegetación que puedan favorecer la convivencia vecinal y/ o social. Aunado al hecho de localizarse alejados de otros espacios de confluencia social, no se tiene la opción de construir ciudadanía, ya que se busca la homogenización y no la concordancia entre distintos.

La valoración que se hace sobre el espacio público (calles y banquetas) del sector, respecto a limpieza y buen estado, es considerada como mala, ya que las colindancias con baldíos y ambientes en el límite de la ciudad, en periodos de ventiscas afectan la calidad del aire y del espacio común, aunado a que no existe el servicio de limpieza como en otras ciudades. En este caso, corresponde a los habitantes limpiar el frente de sus casas para contar con este beneficio, condición que no siempre se cumple, ya sea por falta de educación o porque las contingencias ambientales lo impiden.

Los aspectos de habitabilidad urbana relacionados a la participación social o la convivencia entre vecinos no pueden ser mejorados si no se tienen ambientes en común que faciliten el uso del espacio público, si las áreas verdes están abandonadas y si la calidad del espacio para el encuentro social, está alejado o es inexistente. En este sentido la valoración de contar con una propiedad se ve depreciada debido a que el entorno está en malas condiciones, confinando a sus habitantes al espacio arquitectónico, mismo que al ser tan pequeño, pasa a impactar en su apropiación. Apostar a que el 49.1 por ciento aún prioriza que tener su casa es un valor superior a cambiarse de lugar, sin promover ventajas de beneficio, es incrementar las posibilidades de mayor abandono de viviendas, ya que el $51.9 \%$ por ciento aceptaría cambiarse de lugar, ya sea cerca de su familia (, a otra colonia cercana, cerca del centro o se iría a otra. Se observa una gran vulnerabilidad en la situación de deterioro y pérdida de valor en el patrimonio adquirido por gran parte de los residentes.

Se observa que la percepción de habitabilidad urbana en los rubros evaluados infiere que se carece de un entorno habitable debido a las condiciones adversas que ofrece el espacio público, ya sea por deterioro o abandono, ya que los referentes para construir el sentido de pertenencia no los provee la propiedad privada sino el ambiente de armonía, de hacer ciudadanía que se logra mediante el arraigo, la convivencia, la solidaridad y la socialización entre distintos, por lo que la falta de relación entre normatividad vs realidad, son insuficientes para garantizar condiciones de calidad de vida a los habitantes

Se puede concluir que, en Ciudad Juárez los desarrollos habitacionales ubicados en las áreas periurbanas, son espacios que aún se encuentran desarticuladas y distantes de los beneficios, servicios y equipamiento básicos para garantizar el acceso a una ciudad que provee calidad de vida aceptable a sus residentes y que según lo indican o manifiestan los Planes de Desarrollo Urbano e Internacionales en los que México ha suscrito sus compromisos de equidad, se incumplen, teniendo que el derecho a una vivienda digna dista mucho de haberse cumplido.

\section{BIBLIOGRAFÍA}

ABRAMO, Pedro (2010). Mercado y Orden. Del caos a la teoría de la localización residencial. Colombia: Universidad Externado de Colombia

ALCALÁ PALLINI, L. (2007). "Dimensiones urbanas del problema habitacional. El caso de la ciudad de Resistencia, Argentina". Boletín del Instituto de la vivienda INVI, 22 (59), 35-68. 
BAZANT, JAN (2011). "Procesos de desarrollo urbano de las ciudades. Pensar el futuro de México, colección conmemorativa de las revoluciones centurias". En La vivienda popular en México Retos para el siglo XXI. Andrade Narváez, Jorge y Carballo Cruz Everardo (coordinadores). Universidad Autónoma Metropolitana. Primera edición. México.

IMIP, PDU PLAN DE DESARROLLO URBANO. (2009). Instituto Municipal de Investigación y Planeación, Ciudad Juárez, México.

IMIP, PDUS PLAN DE DESARROLLO URBANO SOSTENIBLE. (2016). Instituto Municipal de Investigación y Planeación, Ciudad Juárez, México.

INEGI, (2015). Censo de población y vivienda. México.

LEY DE CATASTRO DEL ESTADO DE CHIHUAHUA. (2008). H. Congreso del Estado, Secretaria de Servicios Jurídicos Legislativos, División de Documentación y Biblioteca. Ley publicada en el Periódico Oficial del Estado No. 92 del 18 de noviembre de 1995. Ultima Reforma POE 2008.12.20/No. 102.

MORENO OLMOS, SILVIA HAYDEÉ. (2008). "La habitabilidad urbana como condición de calidad de vida". Palapa vol. III, núm. II, julio-diciembre, 2008, pp. 47-54 Universidad de Colima. México.

REGLAMENTO DE CONSTRUCCIÓN MUNICIPIO DE CIUDAD JUÁREZ, ESTADO DE CHIHUAHUA (2004), Publicado en el Periódico Oficial del Estado de fecha 09 de octubre del 2004, México.

RUEDA, SALVADOR. (1997). "Habitabilidad y calidad de vida", Biblioteca Ciudades para un futuro más sostenible, Escuela Técnica Superior de Arquitectura de Madrid. Obtenido el 19 de septiembre de 2007: http://habitat.aq.upm.es/cs/p2/a005.htm/ España.

RUEDA, SALVADOR. (1999). "Modelos e indicadores para ciudades más sostenibles". Taller sobre indicadores de huella y calidad ambiental urbana. Departamento de Medi Ambient de la Generalitat de Catalunya.

ZULAICA, LAURA Y CELEMÍN, JUAN PABLO. (2008). "Análisis territorial de las condiciones de habitabilidad en el periurbano de la ciudad de Mar del Plata (Argentina), a partir de la construcción de un índice y de la aplicación de métodos de asociación espacial". Revista de Geografía Norte Grande, 41: 129-146 Argentina. 